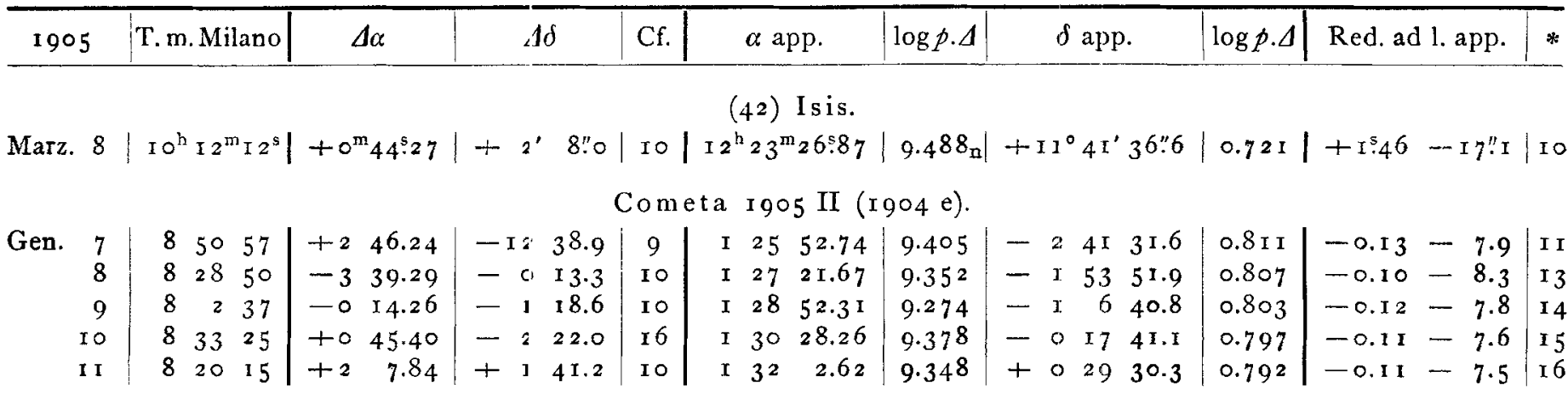

Cometa I904 II $(1904 \mathrm{~d})$.

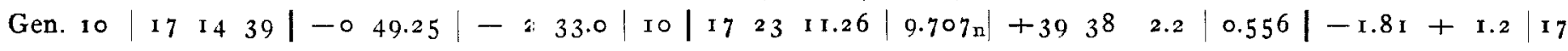

Posizioni medie delle stelle di confronto.

\begin{tabular}{|c|c|c|c|c|c|c|c|c|c|c|}
\hline$*$ & a $1904-05.0$ & $\delta 1904$ & $4-05.0$ & Autorità & $*$ & & 190 & 05.0 & $\delta 1905.0$ & Autorità \\
\hline I & $18^{\mathrm{h}} 17^{\mathrm{m}} 33^{\mathrm{s}} 10$ & $-17^{\circ}$ & $3^{\prime} 53^{\prime \prime} \mathrm{I}$ & \multirow{2}{*}{$\begin{array}{l}\text { AWe. } 443 \text { I } 7 \\
\text { AG. Lind } 352 \text { I }\end{array}$} & 10 & \multicolumn{3}{|c|}{$12^{\mathrm{h}} 22^{\mathrm{m}} 4 \mathrm{I}^{\mathrm{s}} \mathrm{I}_{4}$} & \multirow{2}{*}{$\begin{array}{l}+11^{\circ} 39^{\prime} 45^{\prime \prime} 7 . \\
-\quad 2 \quad 2844.8\end{array}$} & \multirow{4}{*}{$\begin{array}{l}\text { AG. Leipzig I } 4579 \\
\text { BD. }-2: 220 \text { rif. alla } * 12 \\
1 / 3\left(W_{1} 346+2 \operatorname{Rad}_{3} 342\right) \\
\text { AG. Nicolajew } 3 \circ 8\end{array}$} \\
\hline 2 & $64210.5^{8}$ & $+36 \mathrm{I}$ & 69.8 & & I 1 & $\mathbf{I}$ & 23 & 6.63 & & \\
\hline 3 & $6393^{2} \cdot 5^{1}$ & +36 & 713.1 & $\gg \quad \gg \quad 3498$ & 12 & $\mathbf{I}$ & 23 & 10.73 & $-23^{1} 3^{8.8}$ & \\
\hline 4 & $\begin{array}{lll}6 & 37 & 49.30\end{array}$ & $+3^{6} I$ & $2 \quad 12.3$ & 3479 & I 3 & $\mathbf{I}$ & $3 r$ & $\mathbf{I . 0 6}$ & 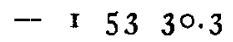 & \\
\hline 5 & $634 \times 6.83$ & +353 & $8 \quad 44 \cdot 5$ & 3429 & 14 & $\mathbf{I}$ & 29 & 6.69 & $\begin{array}{llll}- & 1 & 5 & 14.4\end{array}$ & $\gg \quad>300$ \\
\hline 6 & $\begin{array}{lll}6 & 28 & 19.98\end{array}$ & +354 & 1011.5 & 3377 & 15 & I & 29 & 42.97 & $\begin{array}{llll}-0 & 15 & 11.5\end{array}$ & 302 \\
\hline 7 & 62618.96 & +352 & I 44.4 & $\gg \quad \gg \quad 335^{8}$ & I 6 & $\mathbf{I}$ & 29 & 54.89 & +02756.6 & $3 \circ 3$ \\
\hline 8 & $\begin{array}{lll}7 & 22 & 6.20\end{array}$ & +34 & $8 \quad 7.9$ & AG. Leiden 3 : 39 & 17 & & 24 & $2 \cdot 32$ & +394034.0 & AG. Lund 7 I 44 \\
\hline 9 & $7 \times 8 \quad 49.38$ & +335 & 2.9 & $\gg \quad \Rightarrow \quad 3122$ & & & & & & \\
\hline
\end{tabular}

Le osservazioni furono fatte dal sottoscritto; ad alcuni dei calcoli di riduzione prese parte l'assissente Dottor Giovanni Bottino Barzizza.

Milano, I 905 Marzo 29.

Ingegnere Luigi Gabba.

Osservazioni della cometa 1905 a

fatte col micrometro circolare al rifrattore equatoriale di 8 pollici del R. Osserv. di Brera in Milano.

\begin{tabular}{|c|c|c|c|c|c|c|c|c|c|c|}
\hline 1905 & T.m.Milano & $\Delta \alpha$ & $\angle 1 \delta$ & Cfr. & $\alpha$ app. & $\log p . \Delta$ & $\delta$ app. & $\log p . \Delta$ & Red.ad l. app. & * \\
\hline $\begin{array}{r}\text { Apr. } 6 \\
8\end{array}$ & $\begin{array}{l}9^{\mathrm{h}} \mathrm{I} 5^{\mathrm{m}} 30^{\mathrm{s}} \\
9\end{array}$ & $\begin{array}{l}-\mathrm{I}^{\mathrm{m}} 17^{\mathrm{s}} .84 \\
+0 \mathrm{I} 7.86\end{array}$ & $\begin{array}{ll}+7^{\prime} & 9.1 \\
-13 & 56.6\end{array}$ & $\begin{array}{c}\text { I } 0 \\
9\end{array}$ & 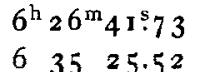 & $\begin{array}{l}9.577 \\
9.577\end{array}$ & $\begin{array}{l}+24^{\circ} \text { × } 5^{\prime} 5^{\prime \prime} .1 \\
+26 \\
2 \text { I } .0\end{array}$ & & $\begin{array}{l}+0.13-7.9 \\
+0.13-6.9\end{array}$ & \\
\hline
\end{tabular}

Posizioni medie delle stelle di confronto.

\begin{tabular}{|c|c|c|c|c|}
\hline$\alpha 1905.0$ & $\delta \mathrm{Ig}$ & 905.0 & & Autorita \\
\hline $6^{\mathrm{h}} 27^{\mathrm{m}} 59^{\mathrm{s}} \cdot 44$ & $+24^{\circ}$ & & $3 " 9$ & riferita alla $* 2$ \\
\hline $6: 7 \quad 30.21$ & +24 & 64 & 41.2 & (n B 2443 \\
\hline 7.53 & +26 & $46 I$ & I 4.5 & AG. Cambr. 3422 \\
\hline
\end{tabular}

Milano, 1905 Aprile 27.

Luigi Gabba.

\title{
Beobachtungen von Kometen
}

am I 2-zöll. Refraktor, Heidelberg-Königstuhl, astronom. Institut.

\begin{tabular}{l|l|l|l|l|l|l|l|}
\hline Datum & M. Z. Kgst. & $\Delta \alpha$ & $\Delta \delta$ & Vgl. $\mid \mathrm{Bb}$. & $\alpha$ app. & $\log p . \Delta \mid$ & $\delta$ app. $|\log p . \Delta| \mathrm{Red}$. ad l. app. $\mid *$ \\
\hline
\end{tabular}

$19 \circ 5$

Komet 1904 I (1904 a).

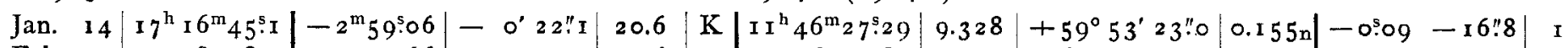

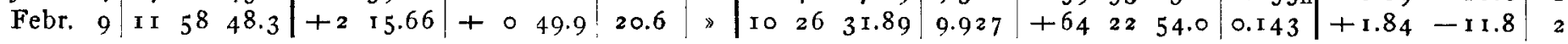

